\title{
Evaluation of the influence of light-curing units on the degree of conversion in depth of a bulk-fill resin
}

\author{
Fernanda-Midori Tsuzuki ${ }^{1}$, Lidiane-Vizioli de Castro-Hoshino ${ }^{2}$, Larissa-Coelho-Pires Lopes ${ }^{3}$, Francielle Sato ${ }^{4}$, \\ Mauro-Luciano Baesso ${ }^{4}$, Raquel-Sano-Suga Terada ${ }^{5}$
}

\author{
${ }^{1}$ Postgraduate student, Department of restorative dentistry, Dental Materials area, Campinas State University (FOP/Unicamp), \\ Piracicaba, Brazil \\ ${ }^{2}$ Postgraduate student, Department of physics, State University of Maringá (UEM), Maringá, Brazil \\ ${ }^{3}$ Postgraduate student, Department of dentistry, State University of Maringá (UEM), Maringá, Brazil \\ ${ }^{4}$ Associate Professor, Department of physics, State University of Maringá (UEM), Maringá, Brazil \\ ${ }^{5}$ Associate Professor, Department of dentistry, State University of Maringá (UEM), Maringá, Brazil
}

\author{
Correspondence: \\ Av. Mandacaru 1550 \\ 87080-000 Maringá \\ PR, Brazil \\ fertsuzuki@gmail.com
}

Received: 07/05/2020

Accepted: 02/07/2020

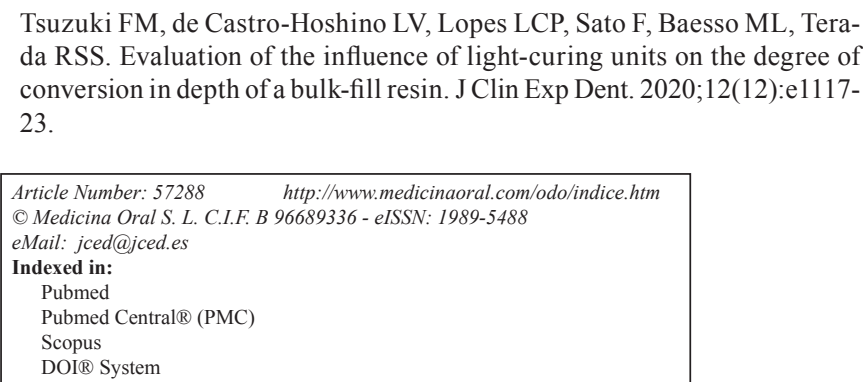

Tsuzuki FM, de Castro-Hoshino LV, Lopes LCP, Sato F, Baesso ML, Terada RSS. Evaluation of the influence of light-curing units on the degree of 23. conversion in depth of a bulk-fill resin. J Clin Exp Dent. 2020;12(12):e1117-

\begin{abstract}
Background: It is known that bulk-fill have been widely studied and used by dentists in the clinic. However, the use of light-curing units that do not have the ability to adequately light-cure these materials at the appropriate depth can affect their clinical performance. The aim of this study was evaluating the influence of 5 different light curing units (LCUs) on the degree of conversion (DC) of a bulk-fill resin at depths of 0 to $4 \mathrm{~mm}$ and determined the effect of using 20s exposure and 40s.

Material and Methods: Cylinders of composite were made in a stainless steel matrix $(n=10)$. The specimens were exposed from the top surface using 5 LCUs: Valo ${ }^{\circledR}$ Cordless (VA); Radii Plus (RA); Emitter.D (EM), Biolux Plus (BI), Woodpecker ${ }^{\circledR}$ (WO). The emission wavelength and the power density was determined. After the photoactivation, the Raman vibrational modes were calculated taking as reference the peaks at 1,601 (aromatic bonds $\mathrm{C}=\mathrm{C}$ ) and $1,640 \mathrm{~cm}^{-1}$ (aliphatic bonds $\mathrm{C}=\mathrm{C}$ ).

Results: The largest difference in DC in 20s, comparing the values obtained in the first and last layer is for BI, with a variation from $61.24 \%$ to $53.86 \%$. Comparing the LCUs, the last layer in $40 \mathrm{~s}$ DC values are $57.40 \%$ (BI), $58.21 \%$ (WO), $58.97 \%$ (VA), $60.90 \%$ (RA) and $62.42 \%$ (EM). The higher the dose $\left(\mathrm{J} / \mathrm{cm}^{2}\right)$ and the close the $\lambda \mathrm{max}$ is to the maximum CQ absorption length $(\lambda \max \sim 470 \mathrm{~nm})$ the better the DC value.

Conclusions: There was a significant difference in the DC values between the LCUs with increasing depth of the bulk-fill increments. Results indicate significant differences in DC among the different LCUs as well as enhanced DC when using 40s exposure compared to 20s. It is suggested that for DC improvement using lower power photoactivator increase the exposure time the exposure time should be $20 \mathrm{~s}$ to $40 \mathrm{~s}$.
\end{abstract}

Key words: Polymerization, Composite Resins, Raman spectroscopy. 


\section{Introduction}

Clinical use of composite resins has become indispensable in dentistry (1). The clinical versatility of resin requires constant improvements in properties for better performance in long-term restorations, which is reflected in the continued launch of new products on the market $(2,3)$. In 2009, bulk-fill resin was launched and the first material commercially available was Surefil ${ }^{\circ}$ SDR resin (Dentsply Caulk, Mildford, DE, USA) (3).

Recent studies show that the bulk-fill resin has improved mechanical properties $(4,5)$, less polymerization stress and reduced microleakage $(6,7)$. Another advantage would be the possibility of filling the cavity in single increments of up to $4 \mathrm{~mm}$, with minimal polymerization shrinkage during the photoactivation process (8), which means reducing the clinical time and reducing the risk of contamination during the restorative procedure (4). To do so, one must take into account the type of LCU used, as this may influence the depth of cure of resin composites. The monomer polymer conversion of the resins is directly related to the intensity, wavelength and irradiation time of the LCU (9).

The degree of conversion (DC) directly affects the physical and mechanical characteristics of the composite resin, influencing the durability of the restoration (10). The presence of unconverted carbon double bonds may render the material more susceptible to degradation, promoting reduced color stability and release of substances with potential for toxicity (11). Therefore, an incorrect photoactivation can cause adverse biological reactions, as well as the reduction of mechanical properties (12). In this way, adequate photoactivation is required so that light-curing resins achieve the properties desired by the manufacturer, a basic requirement for long-term predictable clinical success (13). In addition, with the introduction of different LCUs with increasing power, there is a real danger that dentists are not adequately informed about their use, increasing the number of restorative failures (14).

Among the different devices, the spectral radiant power, the active curing tip diameter and the irradiance are different, thus altering the capacity to light-cure the resins (13). Several studies (15-17) have evaluated the curing depth of bulk fill resins, but none have verified the values to their full extent when using different LCUs.

In this context, the objective of this study was to evaluate the DC in depth of the bulk-fill resin Surefil ${ }^{\circledR}$ SDR with different LCUs. The null hypotheses tested were: (1) DC values do not change significantly with increasing depth of restoration; (2) DC in depth of a bulk-fill resin does not depend on the LCU used for photoactivation of the material and (3) DC values do not change significantly with increasing activation time.

\section{Material and Methods}

-Experimental Design

The DC in depth was evaluated using a bulk-fill flow resin bulk-fill flow, Surefil ${ }^{\circledR}$ SDRTM Flow (Dentsply Caulk, Mildford, DE, USA), photoactivated in the time of 20 and 40 seconds, with 5 types of light-curing units (LCUs): Valo® Cordless/Ultradent (VA); Radii Plus/ SDI (RA); Emitter.D/Schuster (EM), Biolux Plus/Bioart (BI), Woodpecker ${ } /$ Guilin Woodpecker Medical Instrument (WO). The emission wavelength of each LCU was determined by a linear array spectrometer (VS140, HORIBA Jobin Yvon, Kyoto, KA, JPN). The power density of each light-curing unit was also determined (407A, Spectra Physics, Mountain View, CA, USA).

Ten specimens were prepared for each group of LCU and for each time totaling 100 specimens. The DC was evaluated by means of Raman spectroscopy after 24 hours.

-Preparation of specimens

For the preparation of the specimens, we used a stainless-steel matrix containing a central hole measuring $2 \mathrm{~mm}$ in diameter by $4 \mathrm{~mm}$ in height. The matrix was divided in half, with lateral screws, that allow the easy removal of the specimen. The matrix was superimposed on a polyester matrix tape (TDV, Pomerode, SC, BRA) supported on a glass plate and filled with bulk-fill resin. A second polyester tape was placed with light pressure on the matrix in order to avoid excesses, obtain a regular surface and prevent contact with the surface of the light tip (Fig. 1). After the photoactivation at 20 and 40 seconds,

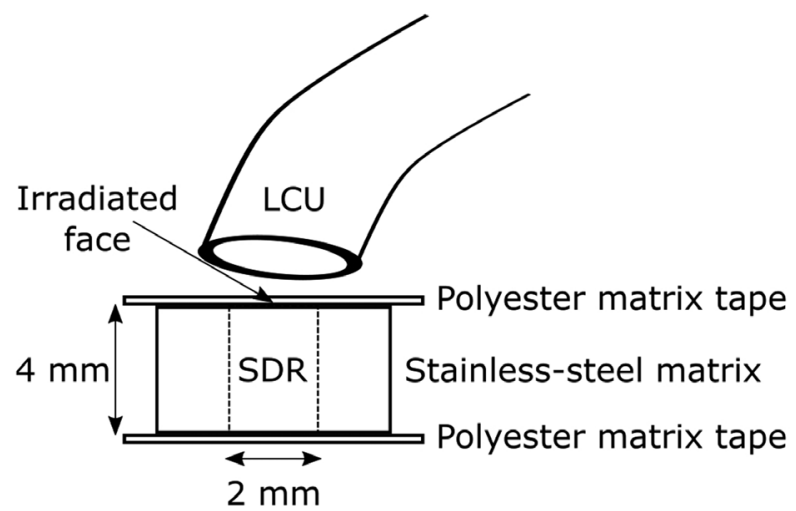

Fig. 1: Schematic representation of specimen preparation for DC evaluation.

each specimen was removed from the matrix and stored in a dry and dark environment at $36^{\circ} \mathrm{C}$ for 24 hours.

-Degree of conversion assessment

All specimens were subjected to DC measurement using a confocal Raman microscope (SENTERRA, Bruker 
Optik GmbH, Ettlingen, BW, DEU), 24 hours after preparation. The measurements were taken with an excitation laser wavelength of $532 \mathrm{~nm}$, nominal power of $20 \mathrm{~mW}$, focused on the specimen by a lens of magnitude of 50x, with 3 seconds of integration time, 20 scans and spectral resolution of 9-15 $\mathrm{cm}^{-1}$ in the region between 1,778-419 $\mathrm{cm}^{-1}$. The spectra were corrected by baseline using OPUS software (7.2, Bruker Optik GmbH, Ettlingen, BW, DEU). Twenty points were read along the length of the specimen, from the face irradiated by the LCU.

-Degree of conversion calculation

In order to evaluate the DC of the bulk-fill resin, the Raman vibrational modes were calculated taking as reference the peaks at 1,601 (aromatic bonds $\mathrm{C}=\mathrm{C}$ ) and $1,640 \mathrm{~cm}^{-1}$ (aliphatic bonds $\mathrm{C}=\mathrm{C}$ ). The percentage of unconverted carbon double bonds $(\% \mathrm{C}=\mathrm{C})$ was determined by the intensity rate of $\mathrm{C}=\mathrm{C}$ bonds $\left(1,601 \mathrm{~cm}^{-1}\right)$ and $\mathrm{C}=\mathrm{C}$ bonds $\left(1,640 \mathrm{~cm}^{-1}\right)$ before and after polymerization. The ratio between the two peaks intensity was calculated for both the polymerized and unpolymerized material. The equation used to calculate the $\mathrm{DC}$ was:

$\mathrm{DC}(\%)=100 \times\left(1-\left(\mathrm{R}_{\text {Polymerized }} / \mathrm{R}_{\text {Unpolymerized }}\right)\right)(1)$

$* \mathrm{R}=$ Ratio between peaks 1,640 and $1,601 \mathrm{~cm}^{-1}$ )

-Statistical analysis

$\mathrm{DC}$ are presented as mean \pm standard deviation of the mean (SD). After tabulation of the results in a database, they were analyzed using analysis of variance (ANOVA). The Shapiro-Wilk test was used to assess the sample normality pattern. The Tukey test was used to compare the different depths and different photoactivation times. The significance level of $p<0.05$.

\section{Results}

The emission spectrum of the LCUs evaluated showed that the devices work at a wavelength of 420 to $500 \mathrm{~nm}$, and for VA there is a peak of light emission in the ultraviolet region (Fig. 2), wavelength region that is required to activate camphorquinone photoinitiator present in the SDR resin (13). The average power rating of each appliance is listed in Table 1.

Figure 3 illustrates the Raman spectra of the SDR resin before and after polymerization for 20 and 40 seconds. Note the polymerization effect by decreasing peak intensity at $1,640 \mathrm{~cm}^{-1}$; this change is associated with the formation of the polymeric structure.

Table 2 presents the significant differences in DC between photoactivators and depths. Comparing the devices, at the time of photoactivation of $20 \mathrm{~s}$, the first depth layer of the resin presented homogeneous DC values for all LCUs. However, in 1-2 mm BI shows the lowest DC values. From $2 \mathrm{~mm}, \mathrm{BI}, \mathrm{WO}$ and RA presented the lowest values. In 40s time, BI showed statistical differences between all photoactivators at all resin depths. In the last layer, DC values in ascending order are $57.40 \%$ (BI), 58.21\% (WO), 58.97\% (VA), 60.90\% (RA) and $62.42 \%$ (EM).

In general, within the same photoactivator, the layers presented statistical differences from $3 \mathrm{~mm}$. The largest difference in DC in 20s, comparing the values obtained in the first and last layer is for BI, with a variation from $61.24 \%$ to $53.86 \%$. In the $40 \mathrm{~s}$, the WO photoactivator is responsible for the greatest variation between the first and last layers, with $64.11 \%$ and $58.21 \%$, respectively.

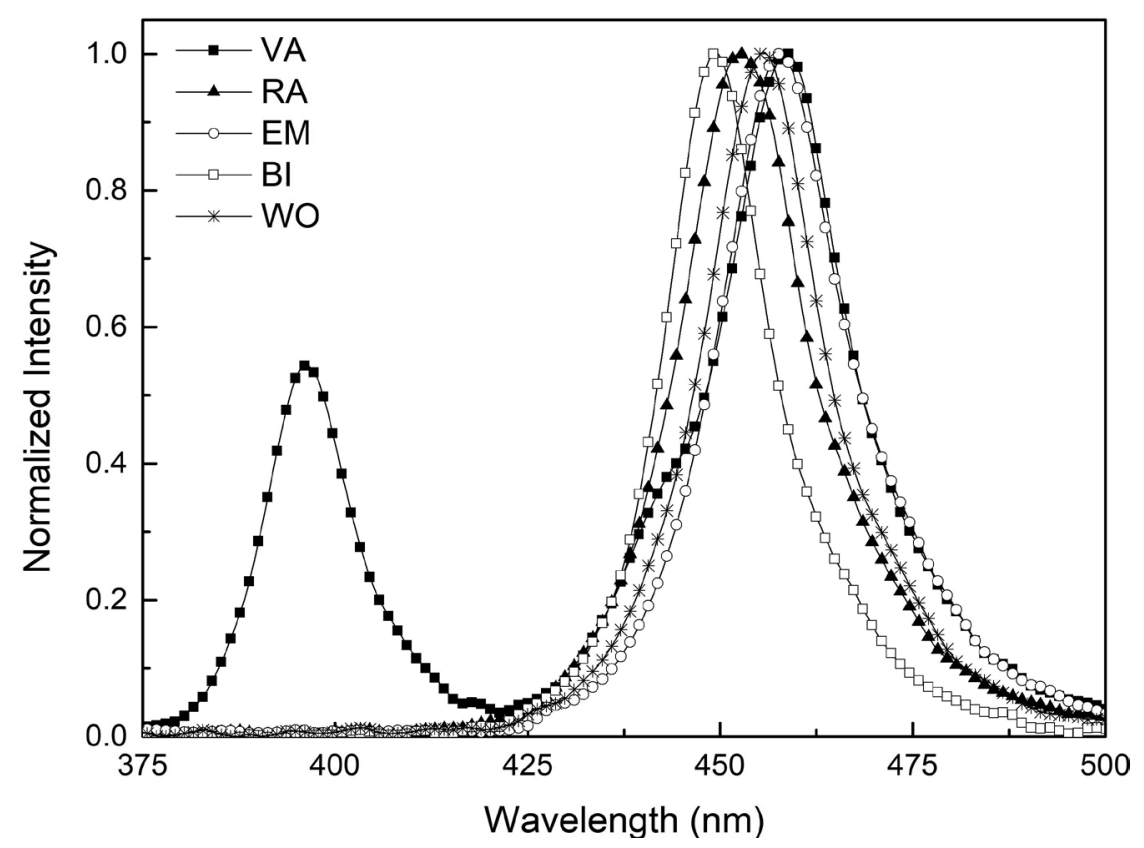

Fig. 2: LCUs emission spectrum. 
Table 1: Specifications of LCUs tested in the study.

\begin{tabular}{|c|c|c|c|c|c|}
\hline $\begin{array}{c}\text { Commercial brand/ } \\
\text { Manufacturer }\end{array}$ & $\begin{array}{c}\text { Wavelength } \\
(\mathbf{n m})\end{array}$ & $\begin{array}{c}\lambda \mathbf{m a x} \\
(\mathbf{n m})\end{array}$ & $\begin{array}{c}\text { Power density } \\
\left(\mathbf{W} / \mathbf{c m}^{\mathbf{2}}\right)\end{array}$ & $\begin{array}{c}\text { LCU dose } \\
\mathbf{2 0 s}\left(\mathbf{J} / \mathbf{c m}^{2}\right)\end{array}$ & $\begin{array}{c}\text { LCU dose } \\
\left(\mathbf{J} / \mathbf{c m}^{2}\right)\end{array}$ \\
\hline Valo $^{\circledR}$ Cordless/Ultradent (VA) & $375-500$ & 458 & 1.01 & 20.2 & 40.4 \\
\hline Raddi Plus/SDI (RA) & $420-495$ & 452 & 1.30 & 26.0 & 52.0 \\
\hline Emitter.D/Schuster (EM) & $420-500$ & 457 & 1.25 & 25.0 & 50.0 \\
\hline Biolux Plus/Bioart (BI) & $420-490$ & 449 & 0.88 & 17.6 & 35.2 \\
\hline $\begin{array}{c}\text { Woodpecker }{ }^{\circledR} / \text { Guilin Woodpecker } \\
\text { Medical Instrument (WO) }\end{array}$ & $420-490$ & 455 & 0.52 & 10.4 & 20.8 \\
\hline
\end{tabular}
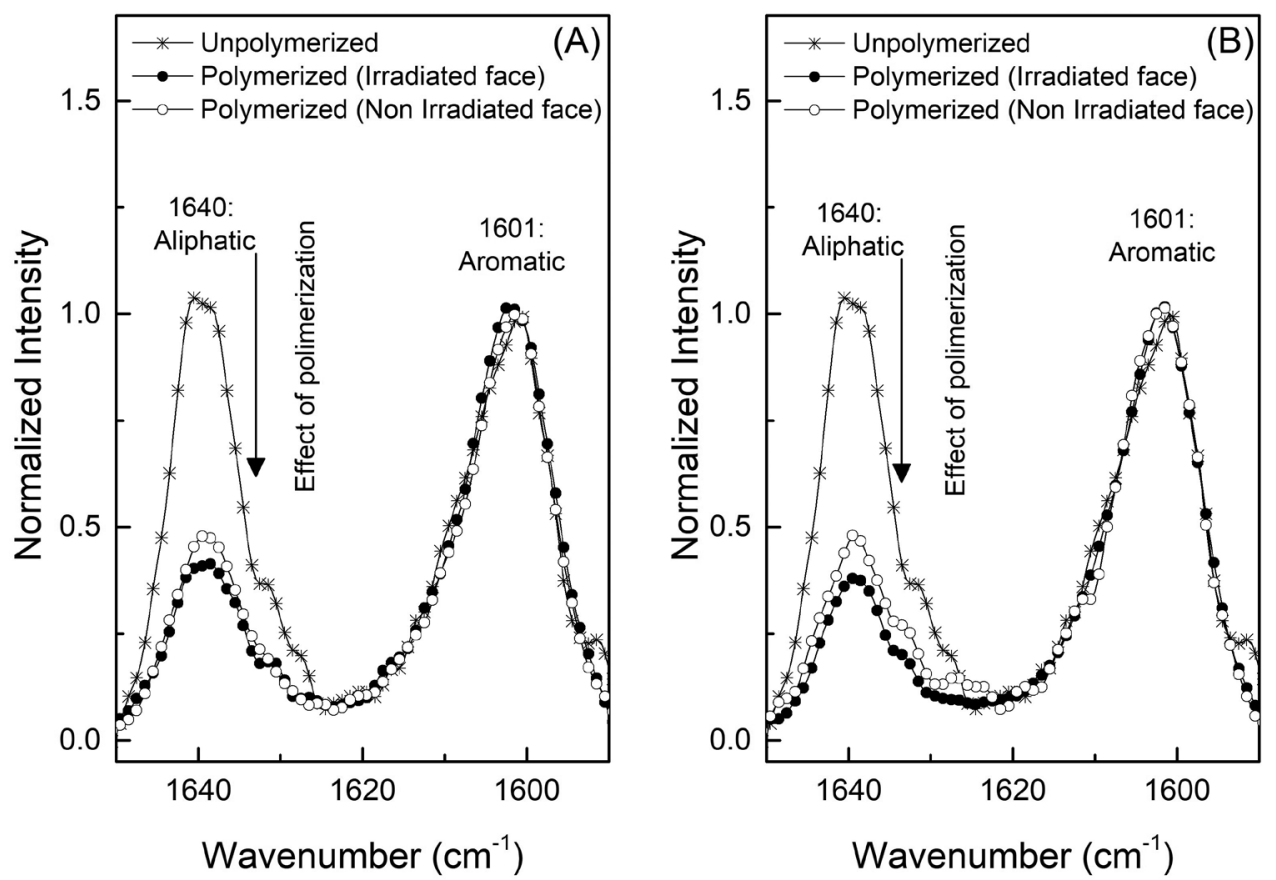

Fig. 3: Peaks of 1,640 $\mathrm{cm}^{-1}$ (aliphatic carbon) and 1,601 $\mathrm{cm}^{-1}$ (aromatic carbon), before and after polymerization along the extension of the specimen. (A) Photoactivated for 20 seconds; (B) Photoactivated for 40 seconds.

Table 2: Mean \pm SD for ranges within $1 \mathrm{~mm}$ thick increments of the degree of conversion (\%) of bulk-fill SDR resin at different depths and different photoactivation times (values measured every $0.2 \mathrm{~mm}$ in figure 4 were summed and the average was obtained for each $\mathrm{mm}$ depth).

\begin{tabular}{|c|c|c|c|c|c|}
\hline \multirow{2}{*}{ Depth (mm) } & VA & RA & EM & BI & WO \\
\cline { 2 - 6 } & \multicolumn{5}{|c|}{$20 \mathrm{~s}$} \\
\hline $0-1$ & $62.6 \pm 0.6 \mathrm{Aa}$ & $61.2 \pm 0.4 \mathrm{Aa}$ & $62.2 \pm 0.9 \mathrm{Aa}$ & $61.2 \pm 0.7 \mathrm{Aa}$ & $61.1 \pm 0.7 \mathrm{Aa}$ \\
\hline $1-2$ & $62.1 \pm 0.7 \mathrm{Aa}$ & $61.2 \pm 0.3 \mathrm{Aa}$ & $62.6 \pm 0.4 \mathrm{Aa}$ & $60.4 \pm 0.3 \mathrm{Ab}$ & $61.0 \pm 0.6 \mathrm{Aa}$ \\
\hline $2-3$ & $60.9 \pm 0.5 \mathrm{Aa}$ & $59.5 \pm 0.7 \mathrm{Bb}$ & $62.1 \pm 0.5 \mathrm{Aa}$ & $57.7 \pm 1.2 \mathrm{Bb}$ & $58.9 \pm 1.3 \mathrm{Bb}$ \\
\hline $3-4$ & $58.9 \pm 1.8 \mathrm{Ba}$ & $56.4 \pm 1.8 \mathrm{Cb}$ & $60.4 \pm 1.4 \mathrm{Aa}$ & $53.9 \pm 2.9 \mathrm{Cb}$ & $56.9 \pm 1.9 \mathrm{Ba}$ \\
\hline & \multicolumn{5}{|c|}{$40 \mathrm{~s}$} \\
\hline $0-1$ & $62.2 \pm 0.7 \mathrm{Aa}$ & $64.3 \pm 1.2 \mathrm{Aa}$ & $64.3 \pm 1.1 \mathrm{Aa}$ & $61.5 \pm 1.3 \mathrm{Ab}$ & $64.1 \pm 0.7 \mathrm{Aa}$ \\
\hline $1-2$ & $63.4 \pm 0.6 \mathrm{Aa}$ & $65.3 \pm 0.5 \mathrm{Aa}$ & $64.5 \pm 0.9 \mathrm{Aa}$ & $60.9 \pm 0.9 \mathrm{Ab}$ & $63.3 \pm 0.9 \mathrm{Aa}$ \\
\hline $2-3$ & $62.3 \pm 0.5 \mathrm{Aa}$ & $64.8 \pm 0.6 \mathrm{Aa}$ & $64.3 \pm 0.8 \mathrm{Aa}$ & $59.7 \pm 0.9 \mathrm{Ab}$ & $61.3 \pm 1.2 \mathrm{Aa}$ \\
\hline $3-4$ & $59.7 \pm 2.6 \mathrm{Ba}$ & $60.9 \pm 4.9 \mathrm{Ba}$ & $62.4 \pm 4.1 \mathrm{Ba}$ & $57.4 \pm 1.9 \mathrm{Ab}$ & $58.2 \pm 2.6 \mathrm{Bb}$ \\
\hline
\end{tabular}

Within their respective times of photoactivation, capital letters compare the columns (DC between different LCUs) and lowercase letters compare lines (DC in depth) $(p<0.05)$. 
Figure 4 shows the significant differences in CG measured every $0.2 \mathrm{~mm}$ at different times. The increase from $20 \mathrm{~s}$ to $40 \mathrm{~s}$ in the photoactivation time of the resin results in the increase of DC, with the exception of the VA photoactivator that maintained the DC similar when comparing the times. The RA obtained the greatest statistical differences comparing the two times of photoactivation. resins, several characteristics have been introduced into their composition $(6,16,19)$, which provides a more uniform conversion of monomers in depth (20-22).

According to the manufacturer, resin has a photosensitive molecule called camphorquinone (CQ) and a new UDMA-based monomer that reduces resin shrinkage stress $(10,23)$. The results showed that the LCUs exhi-

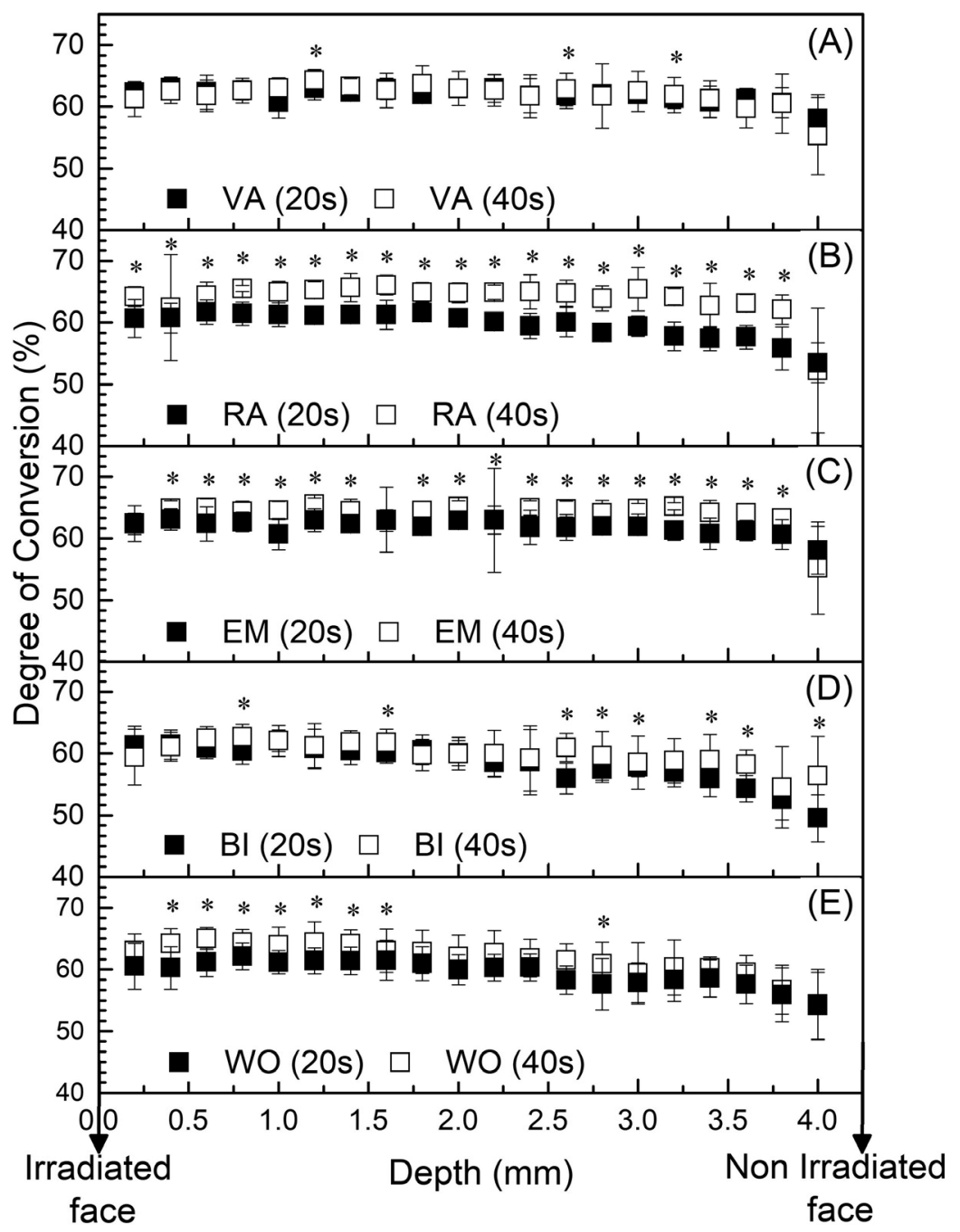

Fig. 4: Mean \pm SD of DC ( $\mathrm{n}=10)$ measured every $0.2 \mathrm{~mm}$ for each LCUs: (A) VA; (B) RA; (C) EM; (D) BI e (E) WO (letter a shows statistical difference between 20 seconds and 40 seconds $p<0,05)$.

\section{Discussion}

The null hypotheses were rejected because the DC values varied significantly according to the increase in the depth of the material, the LCU and the time of photoactivation. As the light absorption of photoinitiators is essential to improve the efficiency of the photochemical reaction, it is important to select resinous compounds with absorption spectra that overlap the emission spectra of the irradiation sources. The literature describes that in order to improve the photoactivation depth of bulk-fill bited 420-490 nm emission peak, coinciding with the maximum absorption peak of the CQ (24).

The emission peak in the ultraviolet region found in Valo (Fig. 2) is because it is a polywave device. For bulk-fill resins containing only $\mathrm{CQ}$ as a photoinitiator, as in the resin case, the monowave and multywave LEDs have shown the same efficiency $(1,24,25)$. For composites containing CQ associated with alternative photoinitiators, the polywave LED has higher DC because these alternative photoinitiators require shorter wavelengths 
(26). Corroborating the literature, it was observed that the ultraviolet peak did not show strong influence on the polymerization of the resin used. However, it is important for the clinician to have a device with two or more emission peaks for use with other resinous materials that have modern photoinitiators (13).

To analyze the DC, different methods have been proposed such as microhardness $(8,20)$, ISO 4049 scraping (27), Fourier transform infrared spectroscopy (FTIR) (5), and vibrational spectroscopy, such as Raman spectroscopy and FTIR, are considered more accurate because they directly quantify the number of unreacted $\mathrm{C}=\mathrm{C}$ bonds $(28,30)$. The main advantage of Raman spectroscopy is to work with the samples in a non-destructive way, which allows multiple measurements in the same sample (10). Differently from previous work evaluating DC only on upper and lower surfaces, this study mapped the DC along the resin by performing a 20-point reading on the entire specimen.

Data show that the LCUs presented differences between them because, although the first layer of resin presented homogeneous DC values for all the LCUs, in the last layer of depth the values between the devices varied from $53.87 \%$ to $60 \%$ in the time of 20 s and from $57.40 \%$ to $62 \%$ within 40 s. To date, the minimum DC for clinically satisfactory restoration has not been established accurately. In the time suggested by the manufacturer of 20 s, the EM and VA devices presented better results values close to $60 \%$, unlike the WO and RA that were close to $56 \%$ and the BI device that presented even lower values. The results suggest that the dose $\left(\mathrm{J} / \mathrm{cm}^{2}\right)$ and the maximum emission wavelength ( $\lambda$ max) of the LCUs influences the DC (Table 1). The higher the dose and the close the $\lambda$ max is to the maximum CQ absorption length $(\lambda \max \sim 470 \mathrm{~nm})$ the better the DC value. The LCUs EM and VA, which presented better results in the time suggested by the manufacturer, have $\lambda \max =457$ and 458 $\mathrm{nm}$, with exposure of 25.0 and $20.2 \mathrm{~J} / \mathrm{cm}^{2}$, respectively. The BI that presented the lowest values has $\lambda \max =449$ $\mathrm{nm}$ with exposure of $17.6 \mathrm{~J} / \mathrm{cm} 2$.

However, the study showed that the increase in photoactivation time may increase DC values. This is because during the activation of the resin, the photons activate the initiator and generate free radicals that start the polymerization, presenting a synergistic effect on the rate of polymerization (26). The literature shows that the optical properties of resin composites and their light activated polymerization reactions are interdependent: greater radiant exposure produces a higher degree of conversion (29). In this way, it is possible to extend the photoactivation time to achieve a higher degree of conversion.

Nevertheless, it is worth emphasizing the limitations of this study. Because it is an in vitro study, the DC may have presented better results because if the restorations were performed clinically, the distance from the cusps, incorrect tip orientation and limitation of mouth opening may interfere with the amount of light that will arrive in the deeper layers (30). In this way, it is necessary for the professional to be sure that the device is in adequate conditions for an effective polymerization (14), and this can be done by routinely monitoring the light output of the LCU through a radiometer (30).

Today, LED-based sources predominate in dental offices (29). Therefore, these results should have an impact on the clinical daily life, since the professional must pay attention to the depth of preparation in which material will be inserted, the photoactivation time of the resin and the type of LCU used, in order to increase the clinical life of restorations and decrease sensitivity, microleakage and caries recurrence.

\section{Conclusions}

In summary, there was a significant difference in the DC values between the LCUs and with increasing depth of the resin increments. It was also observed that the increase in the time of LCUs results in increased DC of the resin. It is suggested that for DC improvement using lower dose photoactivators increase the exposure time from 20 to 40 s.

\section{References}

1. Borgia E, Baron R, Borgia JL. Quality and Survival of Direct Light-Activated Composite Resin Restorations in Posterior Teeth: A 5- to 20-Year Retrospective Longitudinal Study. J Prosthodont. 2019;28:e195-e203.

2. Ferracane JL. Resin composite--state of the art. Dent Mater. 2011;27:29-38

3. Lagocka R, Jakubowska K, Chlubek D, Buczkowska-Radlińska J. Elution study of unreacted TEGDMA from bulk-fill composite (SDR Dentsply) using HPLC. Adv Med Sci. 2015;60:191-8.

4. Leprince JG, Palin WM, Vanacker J, Sabbagh J, Devaux J, Leloup G. Physico-mechanical characteristics of commercially available bulk-fill composites. J Dent. 2014;42:993-1000.

5. Jerri BA. Evaluate polymer degree of conversion of bulk-fill composite restoration. IOSR Journal of Dental and Medical Sciences (IOSR-JDMS) 2015. p. 75-9.

6. Zorzin J, Maier E, Harre S, Fey T, Belli R, Lohbauer U. Bulk-fill resin composites: polymerization properties and extended light curing. Dent Mater. 2015;31:293-301.

7. Kim RJ, Kim YJ, Choi NS, Lee IB. Polymerization shrinkage, modulus, and shrinkage stress related to tooth-restoration interfacial debonding in bulk-fill composites. J Dent. 2015;43:430-9.

8. Jang JH, Park SH, Hwang IN. Polymerization shrinkage and depth of cure of bulk-fill resin composites and highly filled flowable resin. Oper Dent. 2015;40:172-80.

9. Czasch P, Ilie N. In vitro comparison of mechanical properties and degree of cure of bulk fill composites. Clin Oral Investig. 2013;17:22735.

10. Par M, Gamulin O, Marovic D, Klaric E, Tarle Z. Raman spectroscopic assesment of degree of conversion of bulk-fill resin composites--changes at 24 hours post cure. Oper Dent. 2015;40:E92-101.

11. Durner J, Obermaier J, Draenert M, Ilie N. Correlation of the degree of conversion with the amount of elutable substances in nano-hybrid dental composites. Dent Mater. 2012;28:1146-53.

12. Flury S, Hayoz S, Peutzfeldt A, Hüsler J, Lussi A. Depth of cure of resin composites: is the ISO 4049 method suitable for bulk fill materials? Dent Mater. 2012;28:521-8.

13. Price RB, Ferracane JL, Shortall AC. Light-Curing Units: A Review of What We Need to Know. J Dent Res. 2015;94:1179-86. 
14. Leprince JG, Palin WM, Hadis MA, Devaux J, Leloup G. Progress in dimethacrylate-based dental composite technology and curing efficiency. Dent Mater. 2013;29:139-56.

15. Yu P, Yap A, Wang XY. Degree of Conversion and Polymerization Shrinkage of Bulk-Fill Resin Based Composites. Oper Dent. 2017;42:82-9.

16. Rizzante FAP, Duque JA, Duarte MAH, Mondelli RFL, Mendonça G, Ishikiriama SK. Polymerization shrinkage, microhardness and depth of cure of bulk fill resin composites. Dent Mater J. 2019;38:403-10. 17. Tauböck TT, Jäger F, Attin T. Polymerization shrinkage and shrinkage force kinetics of high- and low-viscosity dimethacrylate- and ormocer-based bulk-fill resin composites. Odontology. 2019;107:103-10.

18. Abouelleil H, Pradelle N, Villat C, Attik N, Colon P, Grosgogeat B. Comparison of mechanical properties of a new fiber reinforced composite and bulk filling composites. Restor Dent Endod. 2015;40:262-70. 19. Toh WS, Yap AU, Lim SY. In Vitro Biocompatibility of Contemporary Bulk-fill Composites. Oper Dent. 2015;40:644-52.

20. Alrahlah A, Silikas N, Watts DC. Post-cure depth of cure of bulk fill dental resin-composites. Dent Mater. 2014;30:149-54.

21. Fronza BM, Ayres A, Pacheco RR, Rueggeberg FA, Dias C, Giannini M. Characterization of Inorganic Filler Content, Mechanical Properties, and Light Transmission of Bulk-fill Resin Composites. Oper Dent. 2017;42:445-55.

22. Kim EH, Jung KH, Son SA, Hur B, Kwon YH, Park JK. Effect of resin thickness on the microhardness and optical properties of bulk-fill resin composites. Restor Dent Endod. 2015;40:128-35.

23. Walters NJ, Xia W, Salih V, Ashley PF, Young AM. Poly (propylene glycol) and urethane dimethacrylates improve conversion of dental composites and reveal complexity of cytocompatibility testing. Dent Mater. 2016;32:264-77.

24. Schneider LF, Cavalcante LM, Prahl SA, et al. Curing efficiency of dental resin composites formulated with camphorquinone or trimethylbenzoyl-diphenyl-phosphine oxide. Dent Mater. 2012;28:392-7.

25. Rocha MG, de Oliveira D, Correa IC, Pfeifer CS, Ferracane JL. Light-emitting Diode Beam Profile and Spectral Output Influence on the Degree of Conversion of Bulk Fill Composites. Oper Dent. 2017;42:418-27.

26. Price RB. Light Curing in Dentistry. Dent Clin North Am. 2017;61:751-78.

27. Benetti AR, Havndrup-Pedersen C, Honoré D, Pedersen MK, Pallesen U. Bulk-fill resin composites: polymerization contraction, depth of cure, and gap formation. Oper Dent. 2015;40:190-200.

28. Shin WS, Li XF, Schwartz B, Wunder SL, Baran GR. Determination of the degree of cure of dental resins using Raman and FT-Raman spectroscopy. Dent Mater. 1993;9:317-24.

29. Musanje L, Darvell BW. Polymerization of resin composite restorative materials: exposure reciprocity. Dent Mater. 2003;19:531-41. 30. Rueggeberg FA, Giannini M, Arrais CAG, Price RBT. Light curing in dentistry and clinical implications: a literature review. Braz Oral Res. 2017;31:e61.

Acknowledgement

The authors are thankful to Coordenação de Aperfeiçoamento de Pessoal de Nível Superior (CAPES), Conselho Nacional de Desenvolvimento Científico e Tecnológico (CNPq), Fundação de Amparo à Pesquisa do Estado do Paraná (Fundação Araucária), Financiadora de Estudos e Projetos (FINEP) and Complexo de Centrais de Apoio à Pesquisa (COMCAP).

\section{Author's contributions}

Fernanda Tsuzuki contributed in the collection of data, analysis, interpretation of data and revising

it for critically important intellectual content; Lidiane Castro-Hoshino contributed in the conception,

analysis and interpretation of data; Larissa Lopes contributed in the collection of data, analysis and interpretation of data; Francielle Sato contributed to interpretation of data and revising it for critically important intellectual content; Mauro Baesso contributed in the conception and interpretation of data, drafting the article for critically important intellectual content; Raquel Terada contributed in the conception, analysis and interpretation of data, drafting the article for critically important intellectual content and final approval of the version to be published.

\section{Conflict of interest}

The authors declare that they have no known competing financial interests or personal relationships that could have appeared to influence the work reported in this paper. 